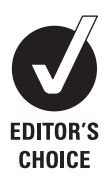

CHOICE

\title{
The risk of myocardial infarction in rheumatoid arthritis and diabetes mellitus: a Danish nationwide cohort study
}

\author{
Jesper Lindhardsen, ${ }^{1}$ Ole Ahlehoff, ${ }^{1}$ Gunnar Hilmar Gislason, ${ }^{1}$ Ole Rintek Madsen, ${ }^{2}$ \\ Jonas Bjerring Olesen, ${ }^{1}$ Christian Torp-Pedersen, ${ }^{1}$ Peter Riis Hansen ${ }^{1}$
}

\section{See Editorial, p 881}

'Department of Cardiology, Gentofte University Hospital, Copenhagen, Denmark 2Department of Internal Medicine - Rheumatology section, Gentofte University Hospital, Copenhagen, Denmark

\section{Correspondence to} Jesper Lindhardsen, Department of Cardiology, section PA (Post 67), Gentofte Hospital, Niels Andersensvej 65, DK-2900 Hellerup, Denmark; jeslin01@geh.regionh.dk

Accepted 23 January 2011 Published Online First 9 March 2011

\begin{abstract}
Objectives To examine in a nationwide cohort whether the risk of myocardial infarction (MI) in patients with rheumatoid arthritis (RA) is comparable to the risk in patients with diabetes mellitus (DM).

Methods The study included the entire Danish population followed from 1 January 1997 until

31 December 2006. Through individual level-linkage of nationwide administrative registers, the authors identified subjects who developed RA and DM. The risk of MI was analysed using multivariable Poisson regression models including data on cardioprotective drugs, comorbidity and socioeconomic status.
\end{abstract}

Results From a total of 4311022 individuals included in the cohort, 10477 and 130215 individuals developed RA and DM respectively. The overall incidence rate ratio (IRR) of $\mathrm{Ml}$ in RA was 1.7 (95\% Cl 1.5 to 1.9), which was similar to the risk in DM (1.7 (1.6 to 1.8); $p=0.64$ for difference). The risk was significantly increased in all groups when stratifying on age and gender, with higher RRs in younger patients. This was especially pronounced in women $<50$ years with $\mathrm{RA}$ or DM, who were subject to a sixfold increase in RR. The RA-related risk of MI was unaffected by the duration of pharmacological RA treatment and corresponded to the overall risk of $\mathrm{MI}$ observed in non-RA subjects, who were on the average 10 years older.

Conclusions RA is associated with the same risk of $\mathrm{Ml}$ as $\mathrm{DM}$, and the risk of $\mathrm{Ml}$ in RA patients generally corresponded to the risk in non-RA subjects 10 years older.

\section{INTRODUCTION}

Patients suffering from rheumatoid arthritis (RA) face an increased risk of cardiovascular disease (CVD). ${ }^{1}$ Several recent studies have shown that the risk of myocardial infarction (MI) is increased in RA with reported RRs of 1.5-2.0 compared to the general population. ${ }^{2-4}$ Traditional cardiovascular risk factors such as hypertension, dyslipidemia, smoking and obesity cannot fully explain the increased risk of MI, and the role of other mechanisms underlying the RA-related risk of CVD remain unclear. ${ }^{2}$ 5-10 These mechanisms include, for example, undertreatment of cardiovascular risk factors in RA patients, adverse effects of pharmacological RA treatment and accelerated atherosclerosis due to inflammatory processes associated with RA. Such mechanisms may also be applicable to other chronic diseases associated with increased risk of CVD, for example, systemic lupus erythematosus, psoriasis and inflammatory bowel disease. ${ }^{11-17}$ Diabetes mellitus is a well-established risk factor for CVD, where specific guidelines for prevention of CVD have been recommended. ${ }^{18}$ From that perspective, it has raised considerable interest, with a cross-sectional and a prospective study of a Dutch cohort of RA and DM patients suggesting that the risk of CVD in individuals with RA is comparable to that of patients with DM. ${ }^{19} 20$ However, in these studies the reported RA and DM cohorts were separated in time by a decade and the studies were not powered to address potential age- and gender-specific risk differences. More insights in this area of research are important to inform discussions on the value of primary prevention of CVD in RA. ${ }^{1}$ Therefore, the present study examined the risk of MI in RA and DM patients within the same nationwide, unselected cohort using unique Danish nationwide registries.

\section{METHODS}

\section{Study cohort and data sources}

The study cohort consisted of all Danish inhabitants alive and aged 16 years or more by 1 January 1997 identified through the Danish Civil Registration system. In Denmark, individual level-linkage of data is made possible by a unique and permanent personal identifier issued at birth, which enabled combination of data from several Danish nationwide registries.

Morbidity was obtained from the National Patient Registry, which contains diagnoses listed according to the International Classification of Diseases (ICD) codes from all hospital admissions (from 1978) and outpatient activities (from 1995) in the form of the 8th revision of the ICD (ICD-8) until 1994 and the 10th revision (ICD-10) thereafter. Dates and institution providing each patient contact were recorded, along with one primary and, if appropriate, one or more secondary diagnoses. Data on medical treatment were obtained from the Danish Register of Medicinal Product Statistics, where all prescriptions dispensed from pharmacies have been recorded from 1995 including information on the Anatomical Therapeutical Chemical (ATC) class, dose, quantity and date of dispensed prescription.

At study baseline (1 January 1997), all subjects were characterised according to concurrent cardiovascular pharmacotherapy defined as dispensed prescriptions during 1996 with $\beta$ blockers (ATC class $\mathrm{C} 07$ ), angiotensin-converting enzyme inhibitors/angiotensin 2 receptor antagonists (C09), statins (C10A), platelet inhibitors (B01AC), loop diuretics (C03C), vitamin $\mathrm{K}$ antagonists (B01AA) 
and spironolactone (C03D). To include the effect of comorbidity, Charlson's comorbidity index was used, modified for use with ICD-10 as previously described. ${ }^{21} 22$ In brief, this index is based on the incidence of 19 prespecified discharge diagnoses 1 year prior to cohort entry. Each of the 19 diagnoses carries a weight from 1 to 6 and the Charlson's comorbidity score is calculated as the resultant weighted sum. An index of socioeconomic status from 0 to 4 was calculated based on the subject income in the years 1992-1996 standardised by age.

\section{Entry criteria and follow-up period}

Subjects with CVD including MI, stroke or coronary revascularisation procedures prior to baseline, as well as subjects with prior RA diagnoses or DM treatment at baseline, were not included in the study cohort.

Subjects were followed until MI, death, emigration or 31 December 2006, whichever came first.

\section{Identification of DM, RA and MI}

During the study period, subjects were monitored for the development of RA, DM and MI. RA patients were identified by ICD codes M5-M6 in combination with a dispensed prescription of disease-modifying antirheumatic drugs (DMARDs), that is, methotrexate (ATC classes L01BA01 and L04AX03), sulfasalazine (A07EC01), azathioprine (L04AX01), hydroxychloroquine (P01BA02), gold therapy (M01CB), leflunomide (L04AA13) and cyclosporine (L04AA01), within a year before or after the time of diagnosis. This method has previously been validated, yielding sensitivity and positive predictive values of $>80 \% .^{23}$ The last of either the prescription date or the date of diagnosis was used as the index date for RA time at risk. Subjects were considered as DM patients at the initiation of pharmacological treatment with oral glucose-lowering drugs or insulin analogues (ACT code A10). ${ }^{24}$ If patients developed both conditions (RA+DM) during the follow-up period, the date of diagnosis of the second of the two conditions denoted the start of time at risk in the models, which included a RA+DM group. MI was detected by the respective ICD-10 codes I21-I22 in the National Patient Registry, as validated previously. ${ }^{25}$

\section{Nested case-control study}

In order to assess the significance of baseline covariates at the time of MI and of the duration of disease, respectively, a nested case-control study was also conducted. All MI cases that occurred in the study period were assigned with up to four gender- and age-matched controls from within the established cohort by random-density sampling. Each case and control was characterised on the date of MI or the MI date of the matched case, respectively, and the presence and duration of RA or DM treatment was determined.

\section{Statistical analysis}

The RA and DM status were considered to be time-dependent and thus contributed to the time at risk in the control group until the RA or DM index date. Incidence rates were presented as events per 1000 person-years at risk.

Multivariable Poisson regression models were fitted to estimate incidence rates ratios (IRR) between controls and RA and DM. Significant effect modification was found for DM and gender, DM and age, RA and age, and RA and DM, and estimates stratified by age and gender as well as overall estimates are presented. Two main models were fitted for analysis: first, the overall risk of MI in RA, DM and RA+DM groups, respectively, was assessed in gender-adjusted and age-adjusted models, and a fully adjusted model accounting for gender, age, calendar year, cardioprotective medication, comorbidity index and socioeconomic index. The second model produced gender-stratified and age-stratified estimates for RA and DM, with adjustment for all other covariates. After stratification, no effect modification between RA and DM was observed. In addition to follow-up

Table 1 Baseline characteristics for the study cohort

\begin{tabular}{|c|c|c|c|c|}
\hline & General population & RA & DM & RA + DM \\
\hline \multicolumn{5}{|l|}{ Participants (n) } \\
\hline Women (\%) & $2046304(51.4)$ & $7011(70.7)$ & $59989(46.3)$ & $337(60.6)$ \\
\hline Men (\%) & $1932517(48.6)$ & $2910(29.3)$ & $69670(53.7)$ & $219(39.4)$ \\
\hline All (\%) & $3978821(100)$ & $9921(100)$ & $129659(100)$ & $556(100)$ \\
\hline \multicolumn{5}{|l|}{ Age (years) } \\
\hline Women & $47.6(19.8)$ & $56.2(15.9)$ & $57.1(18.0)$ & $61.6(12.9)$ \\
\hline Men & $44.6(18.0)$ & $56.8(14.6)$ & $56.4(14.3)$ & $61.3(11.7)$ \\
\hline All & $46.1(19.0)$ & $56.4(15.5)$ & $56.7(16.1)$ & $61.5(12.4)$ \\
\hline \multicolumn{5}{|l|}{ Charlson's comorbidity index } \\
\hline Women & $0.02(0.23)$ & $0.02(0.16)$ & $0.04(0.28)$ & $0.05(0.33)$ \\
\hline Men & $0.02(0.22)$ & $0.02(0.16)$ & $0.04(0.28)$ & $0.03(0.22)$ \\
\hline All & $0.02(0.22)$ & $0.02(0.16)$ & $0.04(0.28)$ & $0.04(0.29)$ \\
\hline \multicolumn{5}{|l|}{ Socioeconomic index } \\
\hline Women & $1.60(1.29)$ & $1.48(1.30)$ & $1.31(1.26)$ & $1.20(1.22)$ \\
\hline Men & $2.45(1.40)$ & $2.49(1.33)$ & $2.27(1.41)$ & $2.29(1.39)$ \\
\hline All & $2.01(1.41)$ & $1.78(1.39)$ & $1.83(1.43)$ & $1.63(1.40)$ \\
\hline \multicolumn{5}{|c|}{ Baseline cardioprotective therapy (n) } \\
\hline$\beta$ blocker (\%) & $126636(3.2)$ & $516(5.2)$ & $11212(8.6)$ & $54(9.7)$ \\
\hline Statin $(\%)$ & $17136(0.4)$ & $71(0.7)$ & $1978(1.5)$ & $9(1.6)$ \\
\hline ACEI/ARB (\%) & $88927(2.2)$ & $392(4.0)$ & $10384(8.0)$ & $47(8.5)$ \\
\hline Loop diuretic (\%) & $106371(2.7)$ & $420(4.2)$ & $9662(7.4)$ & $58(10.4)$ \\
\hline Spironolactone (\%) & $12247(0.3)$ & $45(0.5)$ & $1335(1.0)$ & $8(1.4)$ \\
\hline Vitamin K antagonist (\%) & $17471(0.4)$ & $93(0.9)$ & $1630(1.3)$ & $15(2.7)$ \\
\hline Platelet inhibitor (\%) & $117116(2.9)$ & $438(4.4)$ & $8976(6.9)$ & $48(8.6)$ \\
\hline
\end{tabular}

Values stated as means (SD) unless otherwise indicated.

$\mathrm{ACEl}$, angiotensin-converting enzyme inhibitors; ARB, angiotensin 2 receptor blockers; DM, diabetes mellitus; IRR, incidence rate ratio; $\mathrm{RA}$, rheumatoid arthritis. 
Table 2 Incidence rates and adjusted incidence rate ratios (IRRs) of myocardial infarction from Poisson regression analysis (overall estimates)

\begin{tabular}{|c|c|c|c|c|c|}
\hline & General population & $\mathbf{R A}$ & DM & $\mathrm{RA}+\mathrm{DM}$ & $\begin{array}{l}\text { Test for difference RA } \\
\text { versus } \mathrm{DM}^{*}\end{array}$ \\
\hline Time at risk (1000 person-years) & 37139 & 46 & 489 & 1.6 & \\
\hline Crude incidence $(\mathrm{n} / 1000$ person-years $(\mathrm{CI}))$ & $2.0(2.0$ to 2.1$)$ & $5.7(5.1$ to 6.5$)$ & 8.1 (7.8 to 8.3$)$ & $13.0(8.3$ to 20.0$)$ & \\
\hline \multicolumn{6}{|l|}{ Risk of myocardial infarction (IRR (CI)) } \\
\hline Overall risk, gender-adjusted and age-adjusted & Reference & $\begin{array}{l}1.7(1.5 \text { to } 1.9) \\
p<0.001\end{array}$ & $\begin{array}{l}1.9(1.8 \text { to } 2.0) \\
p<0.001\end{array}$ & $\begin{array}{l}2.9(1.9 \text { to } 4.4) \\
p<0.001\end{array}$ & $p=0.06$ \\
\hline
\end{tabular}

${ }^{*}$ Wald $\chi^{2}$ test for difference of RA and DM IRR estimates.

${ }^{\dagger}$ After entry into disease-specific group.

${ }^{\ddagger}$ Adjusted for gender, age, calendar year, cardioprotective medication, comorbidity and socioeconomic status.

DM, diabetes mellitus; RA, rheumatoid arthritis.

time, age (overall model) and calendar year (overall and stratified models) were included as timescales.

Conditional logistic regression models were used to obtain odds ratios for RA and DM exposure in the supplementary nested case-control study.

Wald $\chi^{2}$ tests were used to evaluate difference between parameter estimates within the models. A two-tailed $\mathrm{p}$ value less than 0.05 was considered significant and 95\% CI were also reported. SAS version 9.1 was used for data management and Stata version 11.0 for statistical analysis.

\section{Ethics}

This study was approved by The Danish Data Protection Agency (ref. 2007-41-1667). Retrospective registry studies do not require ethical approval in Denmark and all available data were encrypted to ensure full anonymity. The study was conducted and reported in accordance with the Strengthening the Reporting of Observational Studies in Epidemiology (STROBE) recommendations. ${ }^{26}$ The authors had full access to the data and take full responsibility for its integrity.

\section{RESULTS}

\section{Baseline characteristics}

A total of 4311022 subjects, aged 16 years or more on 1 January 1997, were identified. Due to prior disease (CVD n=102 229; RA $\mathrm{n}=19$ 377; DM n=79 674) and incomplete data entries ( $\mathrm{n}=1821)$, 192065 subjects were excluded from the analysis. During the 10-year study period, 9921 subjects developed RA, 129659 developed DM and 556 developed both conditions. Baseline characteristics of the study population are summarised in table 1. As expected, the reference population was, in general, younger, healthier and had a higher socioeconomic status than patient groups. Compared to DM patients, RA patients were more often women, used less cardioprotective medications and had less comorbidity, whereas age was similar in the two groups.

\section{Overall risk of $\mathrm{MI}$}

The number of MIs, total time at risk, mean follow-up and IRRs are listed in table 2. The mean follow-up in the RA, DM and RA+DM groups were 4.6, 3.9 and 3.0 years respectively. Disregarding baseline differences between groups, the crude incidence rates of MI were higher in RA ( $\approx$ threefold increase), DM ( $\approx$ fourfold increase), and RA and DM ( $\approx$ sixfold increase) compared to the general population.

Poisson regression analysis with adjustment only for age and gender showed a 1.7 (95\% CI 1.5 to 1.9) increased IRR of MI in RA patients compared to the general population, which was similar to the IRR of 1.9 (1.8 to 2.0) in DM patients (table 2). In agreement with the distribution of baseline characteristics, the risk estimate in DM patients was reduced to 1.7 (1.6 to 1.8) in the fully adjusted model, which was not different from the risk conferred by RA 1.7 (1.5 to 1.9, p=0.64 for difference between risk estimates), whereas the risk in RA patients was unaffected by these adjustments. In patients who suffered from RA and DM the IRR was 2.6 , which roughly equalled the predicted additive risk of the two separate diseases.

\section{Effects of age and gender}

When subjects were stratified by gender, the MI risk estimates for RA patients did not differ between women (IRR 1.7 (1.4 to 2.0)) and men (1.6 (1.4 to 1.9); $\mathrm{p}=0.78$ for difference), whereas in DM patients, women (2.0 (1.9 to 2.1$)$ ) were at higher risk than men (1.5 (1.5 to 1.6); $\mathrm{p}<0.001$ for difference).

The gender-stratified and age-stratified analysis demonstrated a significantly higher RR of MI in all age strata for RA and DM patients, with a notable decline in IRR with age (table 3). Among women with RA, the risk of $\mathrm{MI}$ in the youngest age group was 5.5 times the risk in the reference group, which was comparable to the 5.9-fold increased RR of MI found in DM patients in this age stratum. Although the RR was markedly raised in women with RA between 50 and 65 years (IRR 1.7), it was significantly lower than for DM patients (IRR 2.6), whereas the risks of MI were fully comparable between RA and DM in the oldest age group of women. The pattern of RR in RA compared to DM was different in men, where the IRRs in the two oldest age groups were comparable, and even tended to be slightly higher in the 50-65 years age group of RA patients compared to same-aged DM patients (IRR 2.0 vs $1.5 ; \mathrm{p}=0.06$ ). Although the youngest RA men also had a markedly raised IRR, DM patients in this age stratum had a significantly higher RR (IRR 2.1 vs 4.9; $\mathrm{p}=0.02$ ).

For RA and DM patients the absolute rate difference, that is, the excess number of MIs per 1000 person-years associated with the disease compared to the general population, increased markedly with age and was higher for men than women (table 3).

To ascertain the impact of age on the risk of MI in RA patients, event rates and IRRs were calculated according to 10-year subject age intervals for RA patients and control subjects (figures 1 and 2). The crude risk rates and results from the fully adjusted regression model showed that $\mathrm{RA}$ patients had the same, or higher, risk of $\mathrm{MI}$ as control subjects, who were on average 10 years older.

\section{Additional analyses}

In order to exclude any differential effect between type 1 and type 2 DM patients, the analyses were repeated excluding 
Table 3 Incidence rates, rate differences and adjusted incidence rate ratios (IRR) of myocardial infarction (MI)

\begin{tabular}{|c|c|c|c|c|c|c|c|c|c|c|c|c|c|}
\hline & \multicolumn{3}{|c|}{ General population } & \multicolumn{5}{|c|}{ Rheumatoid arthritis } & \multicolumn{5}{|c|}{ Diabetes mellitus } \\
\hline & $\begin{array}{l}\text { MI cases } \\
\text { (n) }\end{array}$ & $\begin{array}{l}\text { Time at } \\
\text { risk } \\
\text { (1000 py) }\end{array}$ & $\begin{array}{l}\text { Incidence } \\
\text { Rate }\end{array}$ & $\begin{array}{l}\text { MI cases } \\
\text { (n) }\end{array}$ & $\begin{array}{l}\text { Time at } \\
\text { risk } \\
\text { (1000 py) }\end{array}$ & $\begin{array}{l}\text { Incidence } \\
\text { rate }\end{array}$ & $\begin{array}{l}\text { Rate } \\
\text { difference }\end{array}$ & IRR (CI) & $\begin{array}{l}\text { MI cases } \\
\text { (n) }\end{array}$ & $\begin{array}{l}\text { Time at } \\
\text { risk } \\
(1,000 \mathrm{py})\end{array}$ & $\begin{array}{l}\text { Incidence } \\
\text { rate }\end{array}$ & $\begin{array}{l}\text { Rate } \\
\text { difference }\end{array}$ & IRR (CI) \\
\hline Less than 50 & 2531 & 12033 & 0.2 & 15 & 11.0 & 1.4 & 1.2 & $\begin{array}{l}5.5 \\
\text { (3.3 to } 9.2 \text { ) }\end{array}$ & 91 & 64.8 & 1.4 & 1.2 & $\begin{array}{l}5.9 \\
\text { (4.8 to } 7.3 \text { ) }\end{array}$ \\
\hline $50-65$ & 6777 & 4113 & 1.6 & 33 & 11.9 & 2.8 & 1.2 & $\begin{array}{l}1.7 \\
\text { (1.2 to } 2.3 \text { ) }\end{array}$ & 388 & 79.3 & 4.9 & 3.3 & $\begin{array}{l}2.6 \\
(2.3 \text { to } 2.9)\end{array}$ \\
\hline \multicolumn{14}{|l|}{ Men (years) } \\
\hline Less than 50 & 9448 & 12371 & 0.7 & 6 & 4.1 & 1.5 & 0.8 & $\begin{array}{l}2.1 \\
\text { (1.0 to } 4.2 \text { ) }\end{array}$ & 342 & 81.5 & 4.2 & 3.5 & $\begin{array}{l}4.9 \\
(4.4 \text { to } 5.5)\end{array}$ \\
\hline $50-65$ & 17006 & 3779 & 4.5 & 50 & 5.3 & 9.4 & 4.9 & $\begin{array}{l}2.0 \\
(1.5 \text { to } 2.6)\end{array}$ & 907 & 113.8 & 8.0 & 3.5 & $\begin{array}{l}1.5 \\
\text { (1.4 to } 1.7)\end{array}$ \\
\hline More than 65 & 20068 & 1842 & 10.9 & 65 & 3.8 & 17.1 & 6.2 & $\begin{array}{l}1.5 \\
\text { (1.2 to } 1.9)\end{array}$ & 1,185 & 67.6 & 17.5 & 6.6 & $\begin{array}{l}1.4 \\
\text { (1.3 to } 1.5)\end{array}$ \\
\hline
\end{tabular}

Results from fully adjusted Poisson regression analysis (stratified by gender and age tertiles).

Incidence rates and rate differences are given as Mls per 1000 person-years.

Py, person-years.

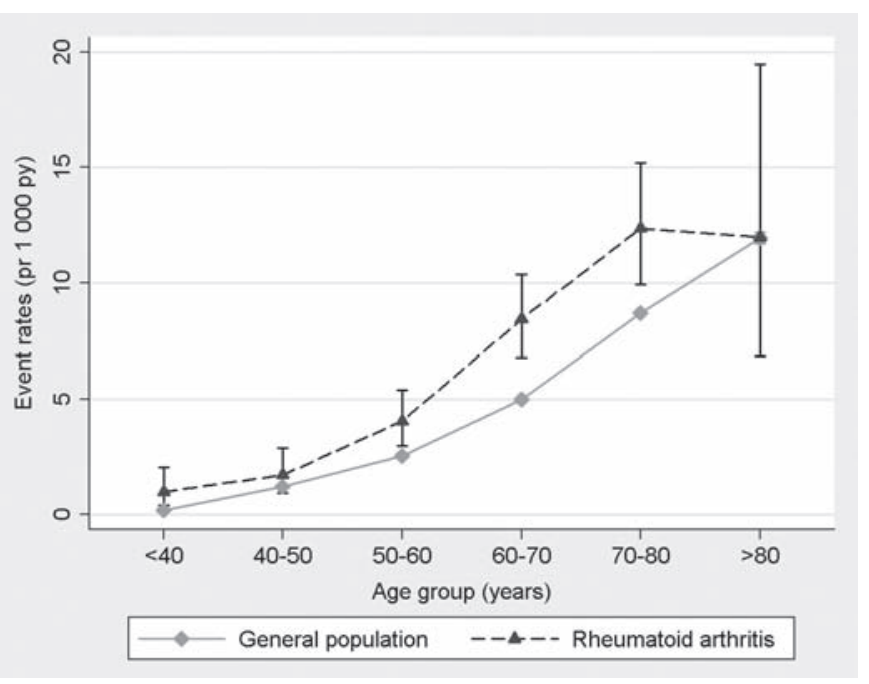

Figure 1 Rates of myocardial infarction in rheumatoid arthritis and in the general population per 1000 person-years (stratified by age). Py, person-years.

subjects with type $1 \mathrm{DM}$, defined by insulin monotherapy as first treatment before 40 years of age $(n=3059)$, and no change in stratified or overall risk estimates was observed. ${ }^{27}$

RA and DM are included in the 19 prespecified diagnoses used in the Charlson's comorbidity index. ${ }^{21}$ This caused DM patients who received a pretreatment DM diagnosis during 1996 to have a relatively higher baseline Charlson index $(n=715)$ than other DM patients. To ensure that this bias did not significantly influence the results, overall and stratified analyses were repeated after subtraction of the excess contribution of DM to the comorbidity index of these patients, as well as after exclusion of subjects with a DM diagnosis (but without glucose-lowering medication) prior to baseline, and no changes were found in the regression analysis estimates (not shown).

The nested case-control study gave results very similar to the cohort study, with odds ratios for RA and DM patients tied at 1.9, when adjusting for current socioeconomic status and comorbidity (table 4). Cardioprotective medications were not included in this analysis as they are routinely prescribed to DM

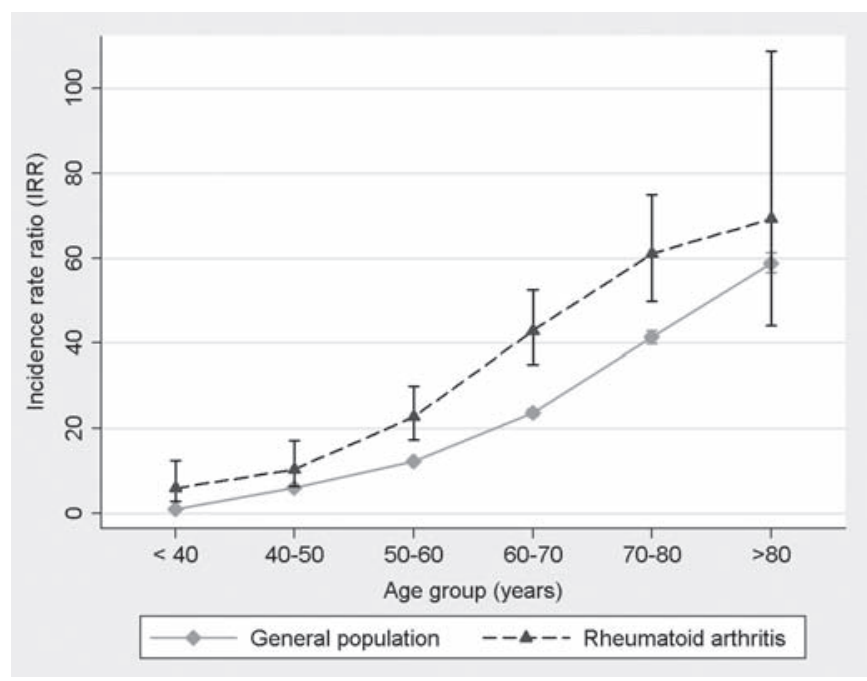

Figure 2 Risk of myocardial infarction in rheumatoid arthritis and in the general population. Results from fully adjusted Poisson regression analysis (stratified by age in 10-year intervals). Reference category: general population age $<40$ years.

patients, that is, their inclusion in the model would invariably lead to underestimation of the impact of DM.

As shown in table 4, the risk of MI was raised significantly in RA and DM regardless of the time elapsed since initiation of pharmacotherapy (treatment duration). Even though there were small differences in IRRs when risk was stratified according to treatment duration, the risk did not increase with time and no clear trend regarding change in risk with treatment duration was observed.

\section{DISCUSSION}

In the current study, the risk of MI in RA patients and DM patients was investigated in an unselected, nationwide cohort followed for a 10-year period. After adjustment for gender, age, calendar year, cardioprotective medication and socioeconomic status, the risk of MI in RA and DM was found to be increased by approximately $70 \%$ compared to the general population. Furthermore, the risk of MI in RA patients was similar to the risk of MI in subjects without RA who were 10 years older. 
Table 4 Risk of myocardial infarction (MI) associated with rheumatoid arthritis and diabetes mellitus. Results from conditional logistic regression analysis in nested case-control study*

\begin{tabular}{|c|c|c|c|}
\hline & MI cases & Controls & $\mathbf{O R}^{*}$ \\
\hline & $n=80104$ & $n=318296$ & (CI) \\
\hline Rheumatoid arthritis & $265(0.33 \%)$ & $603(0.19 \%)$ & 1.9 (1.6 to 2.3$)$ \\
\hline \multicolumn{4}{|c|}{ Treatment duration strata (years) } \\
\hline $0-3$ & 79 (0.10\%) & $216(0.06 \%)$ & 1.7 (1.3 to 2.2$)$ \\
\hline $3-6$ & $105(0.13 \%)$ & $207(0.07 \%)$ & $2.0(1.4$ to 2.9$)$ \\
\hline More than 6 & $81(0.10 \%)$ & $180(0.06 \%)$ & $1.7(1.6$ to 1.9$)$ \\
\hline Diabetes mellitus & 3948 (4.93\%) & 8463 (2.66\%) & $1.9(1.8$ to 1.9$)$ \\
\hline \multicolumn{4}{|c|}{ Treatment duration strata (years) } \\
\hline $0-3$ & $2260(2.82 \%)$ & 4743 (1.49\%) & 2.1 (1.6 to 2.7$)$ \\
\hline $3-6$ & $1208(1.51 \%)$ & 2685 (0.84\%) & 1.9 (1.8 to 2.1$)$ \\
\hline More than 6 & $480(0.60 \%)$ & $1035(0.33 \%)$ & $1.8(1.6$ to 2.1$)$ \\
\hline
\end{tabular}

Values stated are number of Mls (proportion of Mls out of the total number of case or control Mls).

*Adjusted for comorbidity and socioeconomic status (matched on gender and age).

The results also indicated a gender-dependent and age-dependent pattern of MI risk, where the RR was markedly higher in patients under 50 years of age, although men with RA had a more uniform risk profile across age strata.

The comparable risk of $\mathrm{MI}$ in RA and DM was corroborated by the nested case-control study, which also demonstrated that the increased risk of MI in RA patients was independent of disease duration within the timeframe of the current study.

The finding of a $70 \%$ increased risk of $\mathrm{MI}$ in RA patients corresponds well with findings in other studies. ${ }^{2-4} \mathrm{~A}$ recent metaanalysis found the pooled IRR of MI in RA patients to be 2.1, and this slightly higher MI risk compared to the present finding may be explained, in part, by inclusion in the meta-analysis of study populations that were predominantly from the 1980s and early 1990s, when a more conservative approach to RA treatment was the standard of care. ${ }^{28} \mathrm{~A}$ similar sex-dependent and age-dependent pattern of MI risk to observed in the present study has previously been reported by Solomon and coworkers, who also found no overall difference in risk between men and women, and that the highest RR was present in the youngest age groups, especially in women. ${ }^{3}$ The risk attributable to RA also appeared to be high among younger patients in the present study as evaluated by the absolute rate difference in relation to incidence rates. However, because the absolute risk of MI increases markedly with age as illustrated by the increasing incidence rates and absolute rate differences, the potential for prevention of MI in RA patients remains higher in older individuals. Thus, focus on CVD prevention in RA patients should not be reserved for any particular age group. The overall increased risk of CVD in DM patients, as well as the finding that DM confers a higher risk of CVD in women than in men, are well-documented in the literature, including studies from our group indicating that the CVD risk in diabetes patients requiring glucose-lowering therapy is comparable to the risk in non-DM subjects with prior MI. ${ }^{24}{ }^{27}{ }^{29}$ In view of the aggressive approach to primary CVD prevention in DM patients, the recent results from van Halm and coworkers, showing equal prevalence of CVD in two Dutch RA and DM cohorts, have fuelled the discussion on the necessity for more aggressive CVD prevention in RA patients. ${ }^{19}$ Along this line, Stamatelopoulos et al recently found that preclinical atherosclerosis, for example, endothelial dysfunction, increased arterial stiffness and augmented carotidintima thickness, occurred with equal frequency and severity in age-matched, gender-matched and disease duration-matched RA and DM patients. ${ }^{30}$ Although in the Dutch cohort series, subjects were well characterised, RA patients aged younger than 50 and older than 75 years were not included, and RA and DM cohorts were separated in time by a decade. ${ }^{19} 20$ Using the entirely different approach of nationwide registry data analysis, the risk of a single validated end point (MI) in a very high number of patients was evaluated, where selection bias and effects of calendar time were markedly reduced. The present results, therefore, corroborate and clearly expand the existing evidence of a heightened risk of MI in RA patients that is of comparable magnitude to the risk of MI in subjects with DM.

Cardiovascular risk management in patients with RA was recently addressed by the European League Against Rheumatism (EULAR), which in addition to aggressive control of RA activity, recommended annual cardiovascular risk assessment and treatment of identified risk factors according to national guidelines, with adaptation of the risk score models by use of a 1.5 multiplication factor in RA patients with two or more of three criteria, that is, RA duration $\geq 10$ years, rheumatoid factor and/or anticyclic citrullinated peptide positivity, and presence of certain extraarticular RA manifestations. ${ }^{1}$ The present study indicates that this approach may be insufficient in young women with RA, who are subject to a disproportionate CVD risk increase. The suggestion of RA duration $\geq 10$ years as a suitable criterion for increased CVD risk is also debatable, as the risk of $\mathrm{MI}$ in the present study was increased already within the first 3 years of treatment and remained elevated during the observation period of up to 10 years. This phenomenon has also been observed in other cohorts, for example, a Swedish RA inception cohort, where MI risk estimates were the same 1-4 years and 4-12 years from diagnosis. ${ }^{31}$ Certainly, this result does not exclude the possibility of a further increase in CVD risk after 5-10 years of RA, as suggested by the observed differences in risk estimates derived from inception cohorts and established disease cohort, although more intensive therapy aimed at inflammation control in recent years is likely to have reduced the CVD risk increase conferred by RA duration per se. $^{32}$ Along this line, it is also reasonable to expect that the risk of CVD increases before the time of clinical RA diagnosis. ${ }^{33}$

The current data suggest that a reasonable approach to risk assessment in RA other than use of a 1.5 multiplication factor in selected patients as indicated above, may be to add 10 years to the age of the patient and then use the CVD risk score derived from the general population. Indeed, Kremers and coworkers also recently reported that the absolute CVD risk in RA patients was similar to that of non-RA-subjects who were 5-10 years older. ${ }^{34}$ Other patient characteristics, for example, gender, age at disease onset, and markers of disease severity should probably be incorporated in a RA-specific cardiovascular risk prediction model, and development of such a model, its prospective validation and assessment of primary and secondary prevention strategies for cardiovascular disease in RA patients, remain at the centre stage in this area of research.

There are several important limitations to consider when interpreting the present findings. The identification of RA patients relied on dispensed prescriptions and diagnosis rather than the accepted 1987 American College of Rheumatology criteria. ${ }^{35}$ Indeed, this search strategy may have excluded RA patients with putatively 'mild' disease if they were not treated with DMARDs and/or did not attend a hospital clinic. In view of the preferred early introduction of DMARDs and specialist consult advocated by prevailing RA treatment guidelines, this was probably a relatively low number of patients and is unlikely to have influenced the results significantly. It was also not possible to identify DM patients on diet-only treatment, because the use of glucose-lowering drugs was used as proxy for DM. 
Thus in their strictest sense, the present results are only valid for RA patients and DM patients commencing medical treatment.

Although this registry-based approach yielded a very high number of unselected study participants, information was lacking about classical CVD risk factors and some of these, for example, smoking, dyslipidemia and hypertension, may be more prevalent in RA and DM than in the general population. ${ }^{36} 37$ Even though these risk factors to some extent were considered in the analysis by determination of baseline comorbidity and cardioprotective medication, it cannot be ruled out that the inclusion of these clinical variables would have changed the risk estimates. As smoking status was a potentially important factor that could not be determined, the proportion of RA and DM patients with prior admissions for chronic obstructive pulmonary disease at baseline was assessed and only discrete differences were found, which suggests that smoking may not be an important confounder for the comparable risk of $\mathrm{MI}$ in the two diseases (not shown). Also, the contribution of classical risk factors to CVD in RA patients has been estimated to account for approximately half of the risk increase, and as DM patients have a high prevalence of risk factors that contribute to a sizable proportion of CVD risk, it is speculated that inclusion of such variables in the analysis are not likely to considerably decrease the CVD risk estimates for RA patients relative to DM patients. 51037

In conclusion, this nationwide study of approximately 10000 RA patients and $130000 \mathrm{DM}$ patients demonstrated that the risk of MI was similar in RA and DM. Furthermore, it was shown that RA patients had the same risk of MI as control subjects 10 years older, and that the risk of MI remained constant over time after RA treatment commenced. The results corroborate and expand previous findings in this area of research and indicate that patients with RA should be considered for more aggressive primary CVD prevention. Prospective studies of cardiovascular risk management strategies in RA patients are warranted.

Funding The study was supported by an unrestricted grant from the Danish Rheumatism Association.

\section{Competing interests None.}

Ethics approval This study was approved by The Danish Data Protection Agency (ref. 2007-41-1667).

Provenance and peer review Not commissioned; externally peer reviewed.

\section{REFERENCES}

1. Peters MJ, Symmons DP, McCarey D, et al. EULAR evidence-based recommendations for cardiovascular risk management in patients with rheumatoid arthritis and other forms of inflammatory arthritis. Ann Rheum Dis 2010;69:325-31.

2. Turesson C, Jarenros A, Jacobsson L. Increased incidence of cardiovascular disease in patients with rheumatoid arthritis: results from a community based study Ann Rheum Dis 2004;63:952-5.

3. Solomon DH, Goodson NJ, Katz JN, et al. Patterns of cardiovascular risk in rheumatoid arthritis. Ann Rheum Dis 2006;65:1608-12.

4. Wolfe F, Michaud K. The risk of myocardial infarction and pharmacologic and nonpharmacologic myocardial infarction predictors in rheumatoid arthritis: a cohort and nested case-control analysis. Arthritis Rheum 2008;58:2612-21.

5. Gonzalez A, Maradit Kremers H, Crowson CS, et al. Do cardiovascular risk factors confer the same risk for cardiovascular outcomes in rheumatoid arthritis patients as in non-rheumatoid arthritis patients? Ann Rheum Dis 2008;67:64-9.

6. Solomon DH, Curhan GC, Rimm EB, et al. Cardiovascular risk factors in women with and without rheumatoid arthritis. Arthritis Rheum 2004;50:3444-9.

7. Toms TE, Symmons DP, Kitas GD. Dyslipidaemia in rheumatoid arthritis: the role of inflammation, drugs, lifestyle and genetic factors. Curr Vasc Pharmacol 2010;8:301-26.

8. Goodson NJ, Silman AJ, Pattison DJ, et al. Traditional cardiovascular risk factors measured prior to the onset of inflammatory polyarthritis. Rheumatology (Oxford) 2004; $43: 731-6$

9. del Rincón ID, Williams K, Stern MP, et al. High incidence of cardiovascular events in a rheumatoid arthritis cohort not explained by traditional cardiac risk factors. Arthritis Rheum 2001;44:2737-45.
10. Solomon DH, Kremer J, Curtis JR, et al. Explaining the cardiovascular risk associated with rheumatoid arthritis: traditional risk factors versus markers of rheumatoid arthritis severity. Ann Rheum Dis 2010;69:1920-5.

11. Suissa S, Bernatsky S, Hudson M. Antirheumatic drug use and the risk of acute myocardial infarction. Arthritis Rheum 2006;55:531-6.

12. Panoulas VF, Metsios GS, Pace AV, et al. Hypertension in rheumatoid arthritis Rheumatology (Oxford) 2008;47:1286-98.

13. Davis JM 3rd, Maradit Kremers H, Crowson CS, et al. Glucocorticoids and cardiovascular events in rheumatoid arthritis: a population-based cohort study Arthritis Rheum 2007:56:820-30.

14. Libby $\mathbf{P}$. Role of inflammation in atherosclerosis associated with rheumatoid arthritis. Am J Med 2008;121(10 Suppl 1):S21-31.

15. Solomon DH, Avorn J, Katz JN, et al. Immunosuppressive medications and hospitalization for cardiovascular events in patients with rheumatoid arthritis. Arthritis Rheum 2006;54:3790-8.

16. Ha C, Magowan S, Accortt NA, et al. Risk of arterial thrombotic events in inflammatory bowel disease. Am J Gastroenterol 2009;104:1445-51.

17. Ahlehoff $\mathbf{0}$, Gislason $\mathrm{GH}$, Charlot $\mathrm{M}$, et al. Psoriasis is associated with clinically significant cardiovascular risk: a Danish nationwide cohort study. J Intern Med 2010.

18. Buse JB, Ginsberg HN, Bakris GL, et al. Primary prevention of cardiovascular diseases in people with diabetes mellitus: a scientific statement from the American Heart Association and the American Diabetes Association. Circulation 2007;115:114-26.

19. van Halm VP, Peters MJ, Voskuyl $A E$, et al. Rheumatoid arthritis versus diabetes as a risk factor for cardiovascular disease: a cross-sectional study, the CARRE Investigation. Ann Rheum Dis 2009;68:1395-400.

20. Peters MJ, van Halm VP, Voskuyl AE, et al. Does rheumatoid arthritis equal diabetes mellitus as an independent risk factor for cardiovascular disease? A prospective study. Arthritis Rheum 2009;61:1571-9.

21. Charlson ME, Pompei P, Ales KL, et al. A new method of classifying prognostic comorbidity in longitudinal studies: development and validation. J Chronic Dis 1987; 40:373-83.

22. Nuttall M, van der Meulen J, Emberton M. Charlson scores based on ICD-10 administrative data were valid in assessing comorbidity in patients undergoing urological cancer surgery. J Clin Epidemiol 2006;59:265-73.

23. Singh JA, Holmgren AR, Noorbaloochi S. Accuracy of Veterans Administration databases for a diagnosis of rheumatoid arthritis. Arthritis Rheum 2004;51:952-7.

24. Norgaard ML, Andersen SS, Schramm TK, et al. Changes in short- and longterm cardiovascular risk of incident diabetes and incident myocardial infarction-a nationwide study. Diabetologia 2010;53:1612-19.

25. Madsen M, Davidsen M, Rasmussen S, et al. The validity of the diagnosis of acute myocardial infarction in routine statistics: a comparison of mortality and hospital discharge data with the Danish MONICA registry. J Clin Epidemiol 2003:56:124-30.

26. von Elm E, Altman DG, Egger M, et al. Strengthening the Reporting of Observational Studies in Epidemiology (STROBE) statement: guidelines for reporting observational studies. BMJ 2007;335:806-8.

27. Schramm TK, Gislason GH, Køber L, et al. Diabetes patients requiring glucose-lowering therapy and nondiabetics with a prior myocardial infarction carry the same cardiovascular risk: a population study of 3.3 million people. Circulation 2008;117:1945-54.

28. Meune C, Touzé E, Trinquart L, et al. High risk of clinical cardiovascular events in rheumatoid arthritis: Levels of associations of myocardial infarction and stroke through a systematic review and meta-analysis. Arch Cardiovasc Dis 2010;103:253-61.

29. Booth GL, Kapral MK, Fung K, et al. Relation between age and cardiovascular disease in men and women with diabetes compared with non-diabetic people: a populationbased retrospective cohort study. Lancet 2006;368:29-36.

30. Stamatelopoulos KS, Kitas GD, Papamichael CM, et al. Atherosclerosis in rheumatoid arthritis versus diabetes: a comparative study. Arterioscler Thromb Vasc Biol 2009;29:1702-8.

31. Holmqvist ME, Wedrén S, Jacobsson LT, et al. Rapid increase in myocardial infarction risk following diagnosis of rheumatoid arthritis amongst patients diagnosed between 1995 and 2006. J Intern Med 2010;268:578-85.

32. Aviña-Zubieta JA, Choi HK, Sadatsafavi M, et al. Risk of cardiovascular mortality in patients with rheumatoid arthritis: a meta-analysis of observational studies. Arthritis Rheum 2008:59:1690-7.

33. van Halm VP, Nielen MM, Nurmohamed MT, et al. Lipids and inflammation: serial measurements of the lipid profile of blood donors who later developed rheumatoid arthritis. Ann Rheum Dis 2007;66:184-8.

34. Kremers HM, Crowson CS, Therneau TM, et al. High ten-year risk of cardiovascular disease in newly diagnosed rheumatoid arthritis patients: a population-based cohort study. Arthritis Rheum 2008:58:2268-74.

35. Arnett FC, Edworthy SM, Bloch DA, et al. The American Rheumatism Association 1987 revised criteria for the classification of rheumatoid arthritis. Arthritis Rheum 1988; 31:315-24.

36. Boyer JF, Gourraud PA, Cantagrel A, et al. Traditional cardiovascular risk factors in rheumatoid arthritis: A meta-analysis. Joint Bone Spine 2010.

37. Laakso M, Lehto S. Epidemiology of risk factors for cardiovascular disease in diabetes and impaired glucose tolerance. Atherosclerosis 1998;137(Suppl):S65-73. 